\title{
Identification of Ecological Threats, Pressure and Their Relative Severity of Temcha Riverine Forest, North West, Ethiopia
}

\author{
Edeget Merawi \\ Access and Benefit Sharing Directorate, Ethiopian Biodiversity Institute, Addiss Ababa, Ethiopia
}

Email address:

edegetmerawi@gmail.com

\section{To cite this article:}

Edeget Merawi. Identification of Ecological Threats, Pressure and Their Relative Severity of Temcha Riverine Forest, North West, Ethiopia. American Journal of Agriculture and Forestry. Vol. 4, No. 3, 2016, pp. 64-68. doi: 10.11648/j.ajaf.20160403.12

Received: December 8, 2015; Accepted: December 18, 2015; Published: July 4, 2016

\begin{abstract}
The aim of the study was to investigate previous and future potential threats and pressures in the study area. It described the relative severity, coverage, permanence, probability of occurrence and the trend of threats and pressures of the forest. A reconnaissance survey was conducted from October to December 2013 to collect Ecological data. A total of 80 informants were purposively selected from adjacent kebele (the smallest administrative unit in Ethiopia) residents based on their familiarity to threats. Semi structured interview, focus group discussion and guided field walk were used as a tool for data collection. Overgrazing, wood collection, investment expansion and expansion of farmland were the first four sever pressure and threats to the forest. Cutting and clearing, soil erosion, and boundary conflict were given least main concern. The overall mean of multiple pressure trends was increased sharply and wide spread (15-50\%) extent with high impact in the long term (20-100 years) permanence for rehabilitation. The overall mean of multiple threats trend was increased sharply and widespread (15-50\%) extent with high impact in the long-term (20-100 years) permanence for rehabilitation. The result revealed that strong dependency on the forest is leading to aggravation of the identified threats and pressure and deterioration of Temcha Riverine forest. Raising awareness of local communities on the value of forest and ecological consequences of the observed threats is recommended as the possible management techniques.
\end{abstract}

Keywords: Threats, Riverine, Permanence, Extent, Pressure and Rehabilitation

\section{Introduction}

Threats are potential or impending pressure in which a detrimental impact is likely to occur or continue to occur in the future [7]. Pressure are forces, activities or events that have already a detrimental impact on the integrity of the protected area (i.e. that have diminished biological diversity, inhibited regenerative capacity, and/or impoverished the area's natural resources). It includes all legal and illegal activities, and result from direct and indirect impact of an activity [6]. The uncontrolled exploitation of forest areas by anthropogenic factors and depletion of vegetation by natural factors like forest fire has led to the threat as well as the decline in number and area of many plant species [10].

The forest coverage of Amhara National regional State (ANRS) was about $40 \%$ of the total area [2]. However, many areas in the region have been subjected to high human
Population pressure and threats over several years; the soil and other biological resources are extensively used and resulted in decline of productivity [7]. There are still strips of land that are covered with natural vegetation regardless of these exacerbated loss of biophysical resources in many areas of the region. These areas require urgent protection.

East and west Gojjam are the two administrative zones of ANRS, which are mostly known by their crop productivity. Most of the land found in the two zones is suitable and fertile for agriculture and it is highly populated [5]. It is very difficult to see forest areas except farm trees such as Croton macrostachyus and Eucualptus camaldulensis species. The remnant land or uncultivated lands near or around agricultural land or roads are mostly used for grazing and investment purpose.

According to timeline study and key informants around the study area described, the forest and valley area as have been covered with a high density of plant species at the period 
between 1930-1960. However, now a day, intensive use of land for agricultural investment, and high demand of wood for different purposes are becoming a threat to the riverine forest. Thereby, the area is being affected. Based on this background and baseline, the study was designed. The main objective of this study is to investigate the ecological threats, pressures and its relative severity of Temcha riverine forest.

\section{Materials and Method}

\subsection{Description of the Study Area}

\subsubsection{Geographical Location}

Geographically, the forest lies $10^{\circ} 19^{\prime}-10^{\circ} 41^{\prime} \mathrm{N}$ latitude and $37^{\circ} 16^{\prime}-37^{\circ} 45^{\prime} \mathrm{E}$ longitude. In terms of administration, it is shared between of Dembecha district (Western Gojjam) and Machakel district (Eastern Gojjam) (fig. 1) in Amhara Regional State. It is about $345 \mathrm{~km}$ far from the capital city, Addiss Ababa to the North West, $285 \mathrm{~km}$ from the regional city, BahirDar to the South East. The elevation ranges from 950 masl to 2800 masl. The study area is bordered with 14 kebeles (small administration units in Ethiopia) on the side of West and Eastern part of Gojjam and share boundaries with Debrelias District on the South, West Gojjam (Dembecha District) on the West, Bibugn District on the North and Senan District on the North East [9].

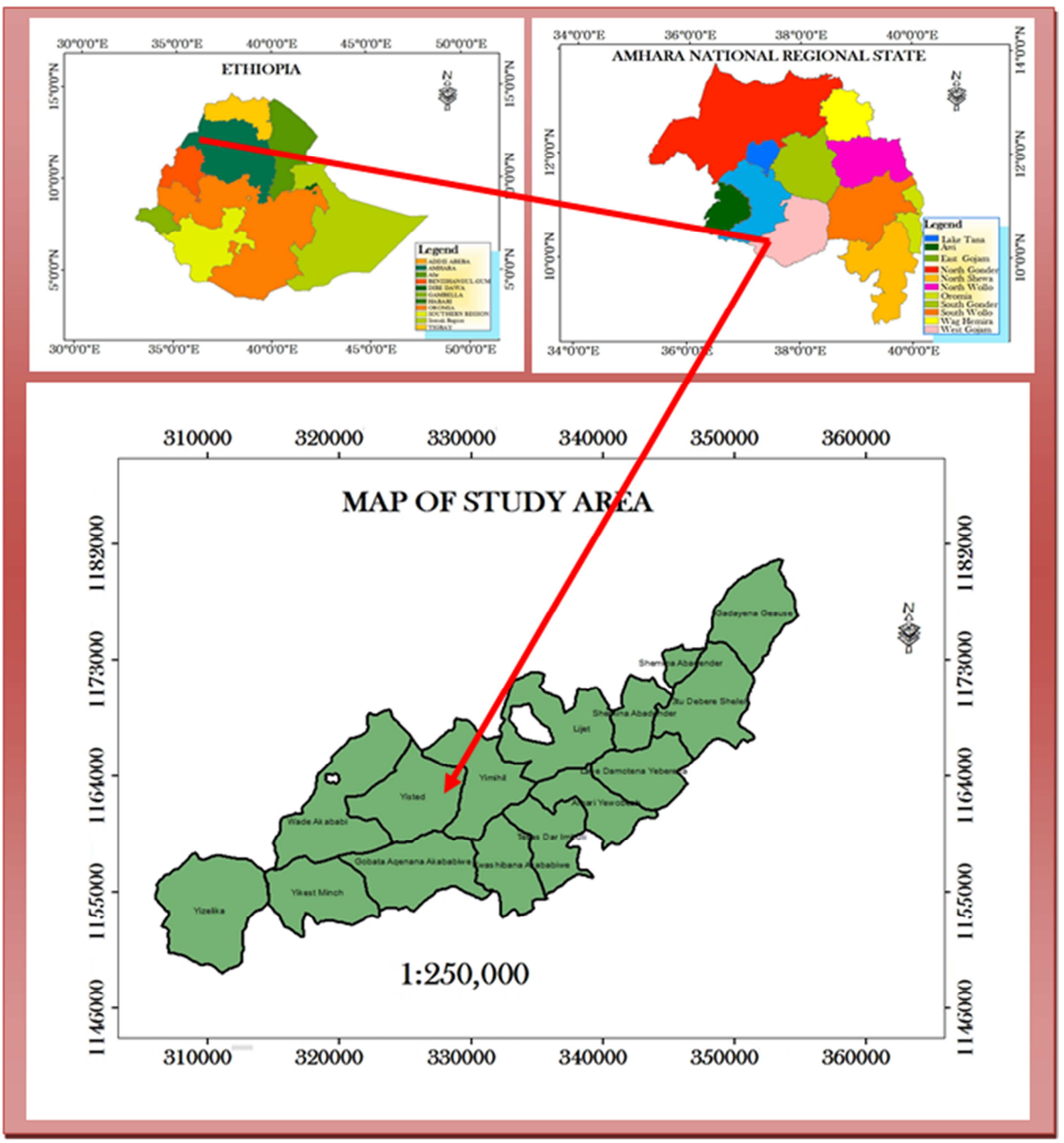

Fig. 1. Map of the study area. 


\subsubsection{Soil}

At a rough scale opinion the soil of the study area is physically reddish brown at the higher altitude while in the lower valley it tends to be dark gray and characteristically high humus and clay content. However, according to [11] (Fig. 3) the study area is diversified soil type, containing vertisols in higher amount.

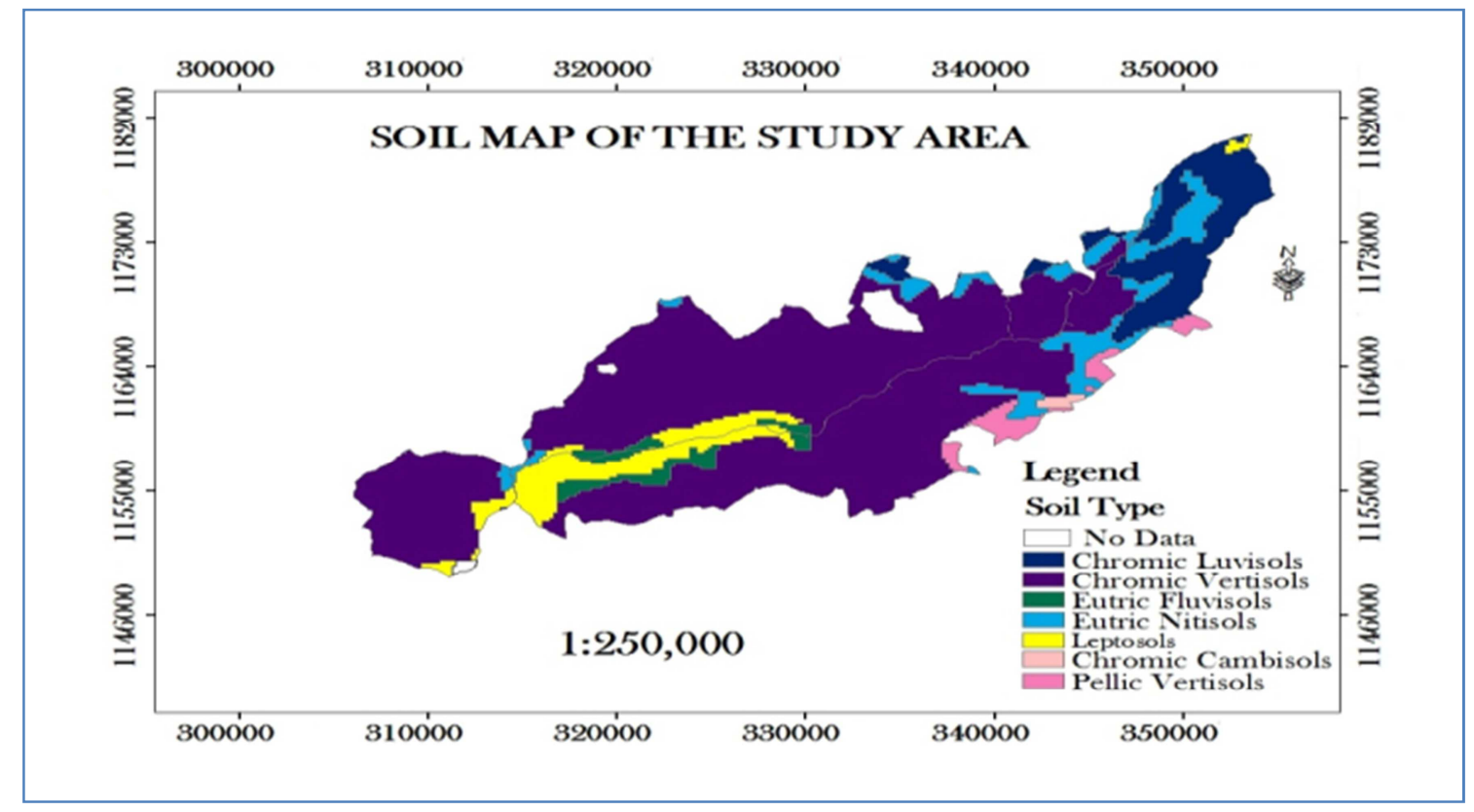

Fig. 2. Soil map of the study area.

\subsection{Research Design}

A reconnaissance survey was conducted from October to December 2013 in selected area of the forest patches. Representative forest patches adjacent to the river were purposively selected based on the availability of threats. Major forest threats and pressure from Lejet Kidane mihret Forest patch (LKFP), Yebuna Forest Patch (YBFP), Abideder Forest Patch (ABFP) and Washa Ameba Tekilehayemanot Monastery Forest Patch (WATMFP) were registered. The most important identified threats of the forest were again screened and sorted based on their effect to the forest (from high to lowest threats and pressure) using Field observation, focus group discussion and semi structured questioner of kebele agricultural officers following [8]. Data were collected on the pressure and threats trend, extent, impact, permanence and degree of pressure in the last five year following [8] (table 1 and 2).

\subsection{Target Population}

The target population of the study was purposively selected local communities, District agricultural officers and kebele agricultural officers. Those local communities having agricultural land near to the riverine forest were purposively targeted.

\subsection{Data Analysis}

The data is analyzed using excel software. The mean score of each threat factor is calculated based on the information obtained from the respondents following [6] measurement scale Whereas, the degree of the pressure and threats occurred in any protected area as a function of extent (E), impact $(\mathrm{I})$ and permanence $(\mathrm{P})$ or degree of pressure $(\mathrm{F})=(\mathrm{E}$ x I x P) [6].

Mean score of each threat factor $=$ Sum of all the score for the particular threat factor / the total number of respondent (80)

The overall mean multiple threat or pressure factor $=$ sum of the mean score of each threat or pressure factor/ total number of threats or pressure for each measurement scale.

Degree of pressure for each pressure factor $(\mathrm{F})=\mathrm{E} \times \mathrm{I}$ x $\mathrm{P}$ where, $\mathrm{E}=$ Extent, $\mathrm{I}=$ Impact and $\mathrm{P}=$ Permanence for each pressure factor

Degree of threat for each threat factor $(\mathrm{F})=\mathrm{E} \times \mathrm{I} \times \mathrm{P}$ where, $\mathrm{E}=$ Extent, $\mathrm{I}=$ Impact and $\mathrm{P}=$ Permanence for each threat factor

Table 1. Pressure analysis standard measurement scale (Trend in the last five years).

\begin{tabular}{|c|c|c|c|c|}
\hline Trend in the past & Extent (E) & Impact (I) & Permanence (P) & Degree o pressure \\
\hline 5-increase sharply & 4-Throughout (>50\%) & 4-severe & 4-permanent (>100 yrs) & 5-severe (49-64) \\
\hline 4-increase slightly & 3-Widespread (15-50\%) & 3-high & 3 -long term (20-100yrs) & 4-high (33-48) \\
\hline 3-remained constant & 2-Scattered $(5-15 \%)$ & 2-moderate & 2-medium (5-20 yrs) & 3 -moderate (17-32) \\
\hline 2-decreased slightly & 1-Localized $(<5 \%)$ & 1-mild & 1-Short term $(<5 y r s)$ & 2-low $(1-16)$ \\
\hline 1-decreased sharply & 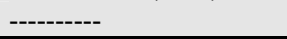 & -------- & 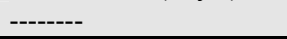 & ------- \\
\hline
\end{tabular}


Table 2. Threat analysis standard measurement scale (probability of occurrence in the future five years).

\begin{tabular}{lllll}
\hline Probability of occurrence & Extent (E) & Impact (I) & Permanence (P) & Degree of threats \\
\hline 5-very high & & & & F= E x I x P \\
4-high & 4-Throughout ( $>50 \%)$ & 4-very high & 4-permanent (>100 yrs) & 5-severe (49-64) \\
3-moderate & 3-Widespread (15-50\%) & 3-high & 3-longterm (20-100 yrs) & 4-high (33-48) \\
2-low & 2-Scattered (5-15\%) & 2-moderate & 2-medium (5-20 yrs) & 3-moderate (17-32) \\
1-very low & 1-Localized (<5\%) & 1-mild & 1-short term (<5yrs) & 1-low (1-16) \\
\hline
\end{tabular}

\section{Result and Discussion}

\subsection{Identification of Threats and Pressure}

A total of eleven threats and pressure were identified during preliminary survey of the four representative forest patches. This are overgrazing, cutting and clearing, wood collection, introduction of alien invasive species, flooding, land fragmentation, investment expansion, soil erosion, boundary conflict, agricultural encroachment and settlement. This finding is agreed with the report Abrham Marye, 2009 done at Bahir Dar Nile River Millennium Park. The most frequently occurred and sever threat were selected and sorted. Based on the inventory and respondent interview seven threats and pressure were given priority to the study forest (table 3). During the threat and pressure inventory of Temcha riverine forest, indicators of human disturbance involvement were observed in most patches. This include the existence of old and large alive trees of some species in the selected forest patches which are found near monasteries and churches and domination of coppiced re growth in most patches of the river. On the bases of this observation or finding wood collection and cutting and clearing were the predominant threats in the last five year in the selected forest patches. This finding (wood collection is predominant threat and pressure) is similar with the respondents' response in which wood collection, overgrazing and investment expansion were the most sever threats and pressures in the last five years. This result showed the local community is inclusively dependent on the forest for multipurpose use.

\subsection{Relative Severity of Threats and Associated Causes}

Responses on pressure and threats were evaluated to investigate the degree of Severity of the threat and associated causes. In the study site seven major pressures and threats mainly due to overuse were identified as major ecological threats. These are overgrazing, wood collection, cutting and clearing, soil erosion, boundary conflict, agricultural encroachment, and investment expansion (fig. 3).

According to the resident's preference overgrazing, wood collection, investment expansion and expansion of farmland were the first four sever pressure and threats to the Riparian forest (fig. 1). This result indicates that, majority of the pressure and threats were occurred mainly due to overuse. Cutting and clearing, soil erosion, and boundary conflict were given least main concern. To see the contribution of each pressure and threats effect following Ervin, 2003 as function of extent (E) coverage), impact (I) (force) and permanence $(\mathrm{P})$ (durability) $\mathrm{F}=(\mathrm{ExIxP})$ were compared (Table 1).

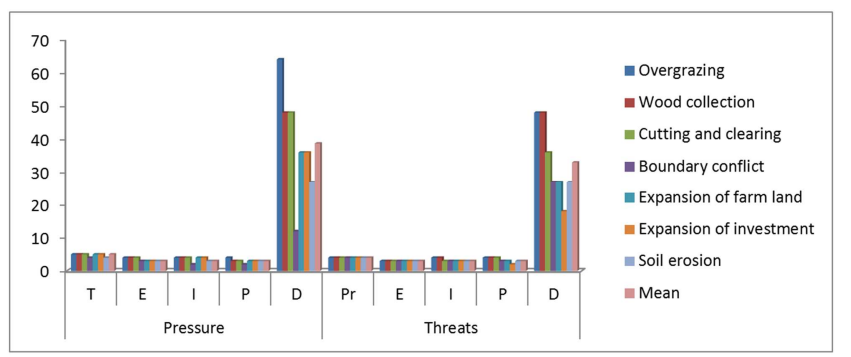

Fig. 3. Contribution of the identified threats and pressures for the disturbance of the forest.

\subsection{Pressure}

The tendency (trend) of the natural and anthropogenic disturbances in the past years were increased sharply in overgrazing, wood collection, cutting and clearing, expansion of farm lands and investment expansion and increase slightly by soil erosion, and boundary conflict (Table 3). The coverage (extent) of disturbances in the past years were throughout $(>50 \%)$ in over grazing, wood collection and cutting and clearing followed by widespread activities $(15-50 \%)$ by soil erosion, boundary conflict, expansion of farmland and investment expansion (Table 3). The forces (impact) of these disturbances in the past years were severe in overgrazing, wood collection, expansion of farmland, investment expansion and cutting and clearing (Table 3), and their durability estimation indicated that overgrazing was the permanent activity ( $>100$ years) followed by long term (20-100 years) in wood collection, soil erosion, cutting and clearing and expansion of farmland as well Medium term of duration (5-20 years) was recorded in boundary conflict. The overall mean (Table 3) multiple pressure trend was increased sharply and wide spread (15$50 \%$ extent) with high impact in the long term (20-100 years) permanence for rehabilitation to its natural state and with moderate to high degree of pressure. 
Table 3. Natural and anthropogenic disturbance activities following (Ervin, 1993) measurement.

\begin{tabular}{|c|c|c|c|c|c|c|c|c|c|c|}
\hline \multirow{2}{*}{ Disturbance activities } & \multicolumn{5}{|c|}{ Pressure } & \multicolumn{5}{|c|}{ Threats } \\
\hline & $\mathbf{T}$ & $\mathbf{E}$ & I & $\mathbf{P}$ & D & Pr & $\mathbf{E}$ & I & $\mathbf{P}$ & D \\
\hline Overgrazing & 5 & 4 & 4 & 4 & 64 & 4 & 3 & 4 & 4 & 48 \\
\hline Wood collection & 5 & 4 & 4 & 3 & 48 & 4 & 3 & 4 & 4 & 48 \\
\hline Cutting and clearing & 5 & 4 & 4 & 3 & 48 & 4 & 3 & 3 & 4 & 36 \\
\hline Boundary conflict & 4 & 3 & 2 & 2 & 12 & 4 & 3 & 3 & 3 & 27 \\
\hline Expansion of farm land & 5 & 3 & 4 & 3 & 36 & 4 & 3 & 3 & 3 & 27 \\
\hline Expansion of investment & 5 & 3 & 4 & 3 & 36 & 4 & 3 & 3 & 2 & 18 \\
\hline Soil erosion & 4 & 3 & 3 & 3 & 27 & 4 & 3 & 3 & 3 & 27 \\
\hline Mean & 5 & 3 & 3 & 3 & 38.7 & 4 & 3 & 3 & 3 & 33 \\
\hline
\end{tabular}

\subsection{Trends}

The probability of occurrence of threats and its coverage (Extent) in the future could be very high and throughout $(>50 \%)$ in all identified natural and human induced activities (Table 1). The force or strength of these disturbances was severe in overgrazing and wood collection followed by high impact in the other identified factors (Table 1). And the durability (permanence) of these activities in the future would be permanent ( $>100$ years) in overgrazing, wood collection and cutting and clearing followed by long term (20-100 years) by the rest activities with the exception of investment expansion a medium term durability (5-20 years) (Table 1). The overall mean (Table 1) of multiple threats trend was increased sharply and widespread (15-50\% extent) with high impact in the long-term (20-100 years) permanence for rehabilitation to its nearly natural state with moderate to high degree of threats potential.

\section{Conclusion and Recommendation}

The pressure and threats in the study area have been prevalent due to driving forces like strong dependency on the forest. All activities of disturbance of the forest are interlinked each other and Therefore, Raising awareness of local communities on the value of forest resources and ecological consequences of major threats, Alternative energy source should be identified and implemented to reduce the dependency on fuel wood or increase the efficiency of fuel wood use, Strengthen threats prevention and mitigation efforts by developing appropriate programs and targeting riverine forest at high risk and Explore individual threats and pressures in greater detail by identifying underlying causes and contributing factors to each activity are recommended as the possible intervention techniques.

\section{References}

[1] Abraham Mary (2009). Diversity, Relative abundance and utilization of woody plants in Bahir Dar Nile River Millennium Park (BDNRMP), Abay (Blue Nile) MSc thesis Submitted to graduate School of Bahir Dar University, Ethiopia.

[2] Cherie Enawgaw, Roman kassahun, Daniel Paulos and Abraham Marye (2006). Report on the Assessment of Alatish Park in Amhara National Reginal State. Federal wildlife Development and Conservation Department, Addis Ababa, Ethiopia.

[3] DDoANRD, (2014). Dembecha District office of Agriculture and Natural Resource Department. Unpublished working Document. Dembecha. Ethiopia.

[4] DDoC, (2014). Dmbecha District office of communication. Unpublished annual statistical report. Dembecha. Ethiopia.

[5] EGZDA, (2014), East Gojjam Zone Department of Agriculture. Unpublished working Document. Debermarkos. Ethiopia.

[6] Ervin, J. (2003). Rapid Assessment and Prioritization of Protected Area Management. WWF Gland, Switzerland.

[7] Fikir Andargie (2010). Floristic composition, structure and threats study of Chemoga forest, Gozamen District, Ethiopia. Unpublished Msc thesis submitted to graduate school of Bahir Dar University, Ethiopia.

[8] Kiringe and Okello. (2007). Threats and their relative severity to wildlife protected areas of Kenya. Applied ecology and environmental research, 5 (2): pp 49-62.

[9] MDDADO, (2014). Machakel and Dembecha District Agricultural Department Office. Unpublished working Document. Machakel and Dembecha, Ethiopia.

[10] Tesfaye Bekele (2002). Plant Population Dynamics of Dodonea angustifolia and Olea europea Sub sp. cuspidata in dry Afromontain Forest of Ethiopia. actaniverstitatis upsaliens Upsala, Sweden.

[11] FAO UNESCO (1974). UNISCO Soil map of the world. UNISCO, Paris. 